\title{
Heterogeneidade na Acessibilidade dos websites das Agências de Viagens: um estudo na Região Centro de Portugal
}

\author{
André Silveiro ${ }^{1}$, Celeste Eusébio², Leonor Teixeira ${ }^{3}$ \\ andresilveiro@ua.pt, celeste.eusebio@ua.pt, lteixeira@ua.pt \\ ${ }^{1}$ DEGEIT; Universidade de Aveiro, 3810-193, Aveiro, Portugal \\ ${ }^{2}$ GOVCOPP/DEGEIT; Universidade de Aveiro, 3810-193, Aveiro, Portugal \\ ${ }^{3}$ IEETA/GOVCOPP/DEGEIT; Universidade de Aveiro, 3810-193, Aveiro, Portugal
}



\begin{abstract}
Resumo: No âmbito do turismo acessível, os websites, enquanto canais promocionais preferenciais, podem representar elementos facilitadores ao acesso físico de produtos e/ou serviços turísticos, caso cumpram com os requisitos de acessibilidade de informação. O presente estudo pretende analisar o nível de acessibilidade dos websites das Agências de Viagens da Região Centro de Portugal, tendo como base as Diretrizes de Acessibilidade do Conteúdo Web (WCAG 2.o) e usando, para o efeito, duas ferramentas automáticas de avaliação (AccessMonitor e TAW online). Os resultados revelam que os websites das Agências de Viagens analisados apresentam alguns problemas em termos de acessibilidade, nomeadamente nos requisitos mais básicos (nível A) das WCAG, e nos princípios 'Percetível' e 'Robusto'. O artigo termina com algumas sugestões para melhorar o nível de acessibilidade dos websites das Agências de Viagens, de forma a que todas as pessoas possam ter acesso à informação, independentemente das suas características e/ou necessidades.
\end{abstract}

Palavras-chave: Agências de Viagens, turismo acessível, pessoas com incapacidade, acessibilidade da web.

\section{Heterogeneity in Accessibility of Travel Agency Websites: A Study in the Central Portugal Region}

Abstract: In the context of accessible tourism, websites, as preferential promotional channels, may facilitate the physical access of tourism products and / or services, if they meet the accessibility requirements of information. This study aims to analyze the level of websites' accessibility of Travel Agency located in the Central Region of portugal, based on the Web Content Accessibility Guidelines (WCAG 2.0) and using two automatic assessment tools (AccessMonitor and TAW online). The results reveal that the Travel Agency websites reviewed have some accessibility issues, mainly in the WCAG basic requirements (level A), and the 'Perceptible' and 'Robust' principles. The article concludes with some suggestions 
for improving the accessibility of Travel Agency websites so that everyone can have access to information regardless of its characteristics and / or needs.

Keywords: Travel Agents, accessible tourism, people with disabilities, web accessibility.

\section{Introdução}

Nos próximos anos espera-se um aumento do número de pessoas com incapacidade (PCI), consequência do envelhecimento da população, do aumento da esperança média de vida, de problemas crónicos de saúde e até mesmo da possibilidade de qualquer pessoa poder vir a adquirir uma incapacidade temporária ou permanente em qualquer momento da sua vida (Gillovic \& McIntosh, 2015). No entanto, apesar das diferenças em termos de motivações e necessidades, as PCI têm o mesmo direito de participar em atividades turísticas. Neste sentido, ao longo dos tempos, têm-se vindo a desenvolver diretivas e regulamentos com o objetivo de existir uma maior inclusão. No caso da indústria do turismo, o esforço na definição dos direitos e deveres de todos os envolvidos no setor, inclusive as PCI, tem vindo a ser notável. A Declaração de Manila (UNWTO, 1980), a Convenção Internacional sobre os Direitos das Pessoas com Deficiência (nomeadamente o artigo 30 ${ }^{\circ}$ ) (UN, 2007) e o Código Mundial de Ética do Turismo (UNWTO, 1999) são exemplos desses esforço, promovendo um turismo mais responsável e inclusivo, através de resoluções que estabelecem o direito a "praticar" turismo, bem como a necessidade de se criarem facilitadores à prática de atividades turísticas de PCI por parte dos fornecedores de produtos turísticos e outros stakeholders (UN, 2007; UNWTO, 1980, 1999).

Apesar de as PCI ainda terem de enfrentar muitas barreiras na área do turismo, temse observado um aumento da oferta de produtos turísticos acessíveis por parte de alguns fornecedores de serviços turísticos. Atualmente começam a existir empresas especializadas na oferta de produtos para este segmento, já que este representa um mercado em crescimento e financeiramente apelativo (Takeda \& Card, 2002; Bowtell, 2015). Também Pinho (2015) realça que este mercado apresenta características diferenciadoras que podem revelar-se numa oportunidade de negócio para as empresas, desde que sejam integradas de forma estratégica.

Porém, para aproveitar o potencial do mercado de PCI, é fundamental apostar no turismo acessível (TA), sendo necessário, portanto, atuar em toda a cadeia de valor (planeamento, compra/aquisição e pós-venda) (Costa, Salvado \& Da Costa, 2014). A esta cadeia de valor pertencem os intermediários em turismo (dos quais fazem parte os Operadores Turísticos - OTs - e as Agências de Viagens - AVs), que são os principais responsáveis pela mediação entre a procura (consumidores) e a oferta (fornecedores) (Lin, Lee \& Chen, 2009).

No entanto, apesar da relevância do mercado de TA, a investigação nesta área, quando comparada com outras áreas de investigação em turismo, é relativamente escassa (Kim e Lehto, 2012). Além disso, dentro da investigação em TA existe uma grande predominância de estudos que analisam o mercado em termos de necessidades, motivações e constrangimentos (ex: Kastenholz, Eusébio \& Figueiredo, 2015; Allan, 2015; Mesquita \& Carneiro, 2016; Melian, Prats \& Coromina, 2016; Kiruthika \& Bindu, 2016; Loi \& Kong, 2017; Lyu, 2017), sendo o número de estudos que analisam os agentes 
responsáveis pela produção e comercialização de produtos turísticos ainda muito escasso (ex: Ozturk, Yayli, \& Yesiltas, 2008; Lin et al, 2009; Schitko \& Losekoot, 2012; Özogul \& Baran, 2016). Dentro deste grupo, o número de estudos que analisa os intermediários em turismo - AVs e OTs - e o TA é ainda mais limitado (Takeda \& Card, 2002; Card, Cole \& Humphrey, 2006).

Este estudo tem como objetivo analisar a acessibilidade dos websites das AVs e OTs existentes na Região Centro de Portugal e, com base nos resultados obtidos, apresentar recomendações que contribuam para o aumento do nível de acessibilidade dos mesmos. No sentido de alcançar o objetivo definido, estruturou-se o presente trabalho em 5 secções. Após uma nota introdutória na presente secção, a secção 2 trata da fundamentação teórica que contextualiza e dá suporte ao trabalho prático, em torno de um conjunto de tópicos como o mercado do TA e a importância das AVs na dinamização desse mercado, bem como aspetos relacionados com a acessibilidade da web. Segue-se a secção 3 que descreve a metodologia adotada para a recolha e análise de dados do estudo empírico. A secção 4 apresenta os resultados do estudo empírico, seguindo-se algumas sugestões para a melhoria da acessibilidade web. Por fim, a secção 5 apresenta as principais conclusões do estudo e as perspetivas de trabalhos futuros.

\section{Revisão da literatura}

\subsection{Mercado do turismo acessível}

A associação entre os termos ‘turismo’ e ‘acessibilidade' surge na Declaração de Manila em 1980 e no relatório ‘Turismo para Todos', em 1989, no Reino Unido. Estes documentos evidenciam a importância de se desenvolver um turismo inclusivo e acessível a todos, impulsionando, assim, um conjunto de eventos e ações que constituem alguns dos pontos históricos do TA. Para além da Declaração de Manila, que reconhece o papel do turismo na qualidade de vida e desenvolvimento humano, destacam-se também o Código Mundial de Ética para o Turismo e a Convenção Internacional sobre os Direitos das PCI, que contribuíram para a criação de políticas e recomendações (direitos e deveres), que visam um setor turístico mais responsável e inclusivo.

$\mathrm{Na}$ verdade, designado muitas vezes também por 'Turismo sem barreiras', 'Turismo Universal', 'Turismo de Acesso' e 'Turismo Inclusivo', o conceito de TA não apresenta um consenso universal nem existe a aceitação de uma definição única (UNWTO, 2016c). Resumidamente, o conceito de TA visa a adaptação dos ambientes, produtos e serviços turísticos de forma a possibilitar o acesso, o uso e aproveitamento, independentemente das capacidades, do estatuto ou condição das pessoas, tendo em conta o Design Universal (UNWTO, 2016c, 2016a).

Todavia, o conceito de TA foi sofrendo alterações ao longo dos últimos, passando a considerar não apenas a acessibilidade para as PCI, mas também acessibilidade para todas as restantes pessoas, com o propósito de uma maior inclusão e, consequentemente, um turismo de qualidade para todos (UNWTO, 2016c).

Em síntese, tanto a UNWTO (2016b) como a comunidade académica utilizam frequentemente a definição deTA proposta por Darcye Dickson(2009), justificando que os autores tentam apresentar um conceito consensual, universal e o mais inclusivo possível. 
Neste trabalho é também adotada a definição de Darcy e Dickson (2009), considerando TA como sendo um turismo que permite a todas as pessoas e, particularmente, às "pessoas com necessidades especiais, incluindo mobilidade, visão, audição e dimensões cognitivas, andarem de forma independente e com equidade e dignidade através da disponibilização de ambientes, serviços e produtos turísticos projetados universalmente, (...) incluindo também pessoas com incapacidades temporárias e permanentes, idosos, obesos, famílias com bebés” (Darcy e Dickson, 2009, p. 34, tradução nossa). Em suma, o TA é um tipo de turismo mais inclusivo e colaborativo que permite às pessoas com necessidades específicas de acesso (incluindo mobilidade, visão, audição e dimensões cognitivas), usufruírem de forma independente e com equidade e dignidade, produtos e serviços turísticos (UNWTO, 2016b).

\subsection{O papel das agências de viagens na dinamização do turismo acessível}

O turismo, para além de ser um setor de grande relevância económica, dinâmico e em crescimento, difere das outras indústrias pela sua multidisciplinaridade e interação com vários outros setores. Também as características dos seus produtos e serviços podem contribuir para que a distribuição na indústria turística seja uma atividade relativamente complexa.

Atualmente, quando se pensa em viajar, começa-se por recolher a informação necessária para o planeamento da viagem, informação essa normalmente dispersa por várias fontes (exemplos: websites oficiais de turismo, blogs, agências de viagens, etc). Posteriormente, o processo de aquisição dos produtos e/ou serviços turísticos, como transporte, alojamento, visitas, pode realizar-se diretamente com cada entidade fornecedora (distribuição direta), ou então pode recorrer-se a uma AV (física ou virtual), ficando esta com o papel da intermediação entre os agentes da oferta e o cliente (distribuição indireta).

Tendo como base as funções e atividades das AVs, estas acabam por ser um "player" preponderante no sistema de distribuição do turismo. Como intermediários, as AVs têm a missão de melhorar e facilitar a coordenação, criando valor no canal de distribuição (Kanellou, 2000; Lee e Cranage, 2017), uma vez que, não só se envolvem no processo de construção, adaptação e transação de um conjunto de serviços (pacotes), como também potenciam aos fornecedores o acesso ao mercado, ao mesmo tempo que disponibilizam ao consumidor um espaço para adquirir serviços de viagem (Lee \& Cranage, 2017).

Kanellou (2000), na sua análise às etapas de intermediação na indústria do turismo, refere que o papel do intermediário tem sido importante ao longo do desenvolvimento e crescimento do setor do turismo. Salienta, ainda, a capacidade destes intermediários se adaptarem às constantes mudanças no turismo, provocadas pelas novas tecnologias de informação e de comunicação (TICs), o que implica, não só alterações do sistema de distribuição, como uma redefinição do próprio papel dos intermediários. De uma forma tradicional, as AVs visam o contato direto com o cliente em instalações físicas e atendimentos presenciais ao balcão. Contudo, derivado do crescimento da indústria turística e do aparecimento de novas TICs, observou-se uma mudança de paradigma neste tipo de negócio (Law, Leung \& Wong, 2004), dando origem a um conjunto de intermediários eletrónicos (eMediaries) no setor do turismo, como são exemplos as agências de viagens online que operam na web. 


\subsection{Acessibilidade da web}

Para que as PCI possam viajar e usufruir das atividades turísticas, alguns autores defendem que a indústria turística deve ter em conta os seguintes requisitos relativamente a este mercado (Buhalis \& Michopoulou, 2011; Michopoulou \& Buhalis, 2013): (i) a acessibilidade física / infraestruturas; (ii) informações sobre a acessibilidade; e (iii) informações acessíveis online (ver Figura 1).

Na verdade, a acessibilidade física acaba por ser o requisito base que todos os operadores turísticos e agências de viagens devem ter em conta, porém, Michopoulou e Buhalis (2013) destacam que, apesar de em muitos países existir legislação que estabelece os requisitos mínimos para assegurar a acessibilidade de edifícios, muitas vezes essa informação não chega às pessoas que dela necessitam para ajudar no planeamento da viagem. É precisamente este especto que compõe o segundo requisito, considerado também fundamental para o mercado do TA (Buhalis \& Michopoulou, 2011), e que diz respeito à disponibilização de informação sobre a acessibilidade. Segundo Michopoulou e Buhalis, (2013), de maneira a providenciar informações sobre a acessibilidade, deve-se ter em conta não só a informação propriamente dita sobre a acessibilidade, mas também o rigor e riqueza dessa informação, bem como o carácter fidedigno da mesma, de modo a atender às necessidades do mercado.

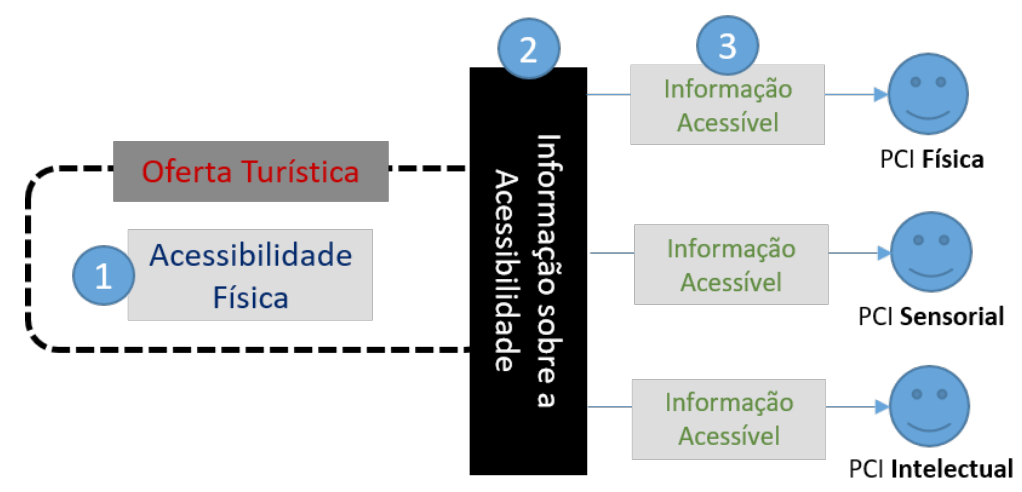

Figura 1 - Diferentes níveis de Acessibilidade.

Por fim, o último requisito para o mercado do TA, referenciado na literatura, está relacionado com a apresentação e disponibilização acessível da informação, particularizando o caso da informação online. Representando a Internet uma das principais fontes de informação aquando do planeamento de uma viagem e o "wordof-mouth online", um dos principais mecanismos de comunicação com maior impacto atualmente, é importante garantir a acessibilidade, quer dos websites, quer da informação apresentada nestes canais de comunicação.

A acessibilidade da Web tem a ver com a capacidade de se aceder à informação sem qualquer tipo de barreira ou obstáculo provocado pela condição do utilizador. Em alguns casos, o nível de acessibilidade depende do tipo de incapacidade/necessidade do ator, e da possibilidade de serem utilizadas tecnologias de apoio, como é o exemplo 
de programas de reconhecimento de voz e sintetizadores de Braille (Vila, González \& Darcy, 2017). Para qualquer situação, os conteúdos Web terão que estar preparados para conectar e comunicar com esse tipo de assistentes.

Neste sentido, foram muitas as recomendações, leis e regulamentos publicados para promover uma maior acessibilidade na Web, porém as recomendações mais importantes e utilizadas são "As Diretrizes de Acessibilidade para o Conteúdo da Web" (WCAG), desenvolvidas pelo World Wide Web Consortium (W3C), que visam apresentar um conjunto de recomendações para ajudar os responsáveis pela construção de websites a tornar o conteúdo mais acessível. Esta ferramenta tem sido utilizada nos mais diferentes tipos de contexto (por exemplo Shi, 2006; Luis, Rocha \& Marcelino, 2017; Mayol, Nadal \& Coll, 2011; Mills, Han \& Clay, 2008; Kuzma, Yen \& Oestreicher, 2009; Carvalho, Lopes, Alexandre \& Alturas, 2016; AkgÜL \& Vatansever, 2016; Vila, González \& Darcy, 2017; Vila, González \& Darcy , 2018).

\section{Análise da acessibilidade dos websites das Agências de Viagens da Região Centro de Portugal}

\subsection{Metodologia}

Com o objetivo de efetuar a análise do nível de acessibilidade dos websites das AVs, utilizou-se um procedimento multi-etapas. Em primeiro lugar, acedeu-se à base de dados do Registo Nacional de Agentes de Viagens e Turismo (RNAVT), sendo que no dia três de janeiro de 2018 encontravam-se inscritos no RNAVT 2557 Agentes de Viagens e Turismo. Com o objetivo de obter o espaço preferencial de presença na Web (website ou facebook) destas AVs e/ou OTs e respectvos endereços Web, foi realizada uma pesquisa no motor de busca Google, através do nome (e da marca) das AVs, do endereço e dos contatos (telefone / telemóvel e e-mail). Para cumprir com os objetivos deste estudo, foram então considerados para análise todas as AVs localizadas na zona Centro de Portugal.

Para analisar a acessibilidade dos websites das AVs foi, então, realizada uma pesquisa para selecionar as ferramentas mais adequadas para a análise. A World Wide Web Consortium (W3C) fornece uma lista de 122 ferramentas (W3C, 2019). No entanto esta análise foi complementada com uma revisão da literatura, por forma a identificar estudos que tentassem resolver a mesma problemática, ou seja avaliação da acessibilidade Web, tendo como base a utilização de ferramentas de avaliação automática (por exemplo Shi,2006; Mills, Han \& Clay, 2008; Kuzma, Yen \& Oestreicher, 2009; AkgÜL \& Vatansever, 2016; Vila, González \& Darcy, 2017; Vila, González \& Darcy, 2018). Foi ainda efetuada uma pesquisa na Web para identificar ferramentas direcionadas para a avaliação da acessibilidade de websites em português. Desta análise, identificou-se um número reduzido de ferramentas potencialmente interessante para a avaliação automática da acessibilidade Web, que após um conjunto de testes e uma extensa análise às ferramentas resultaram em duas: AccessMonitor (http://www.acessibilidade.gov.pt/accessmonitor/) e o 'Test de Accesibilidad Web' (TAW) online (https://www.tawdis.net/).

O AccessMonitor é uma ferramenta automática fornecida pela Fundação para a Ciência e Tecnologia de Portugal, que funciona inteiramente na Web de acordo com as diretrizes de Acessibilidade do Conteúdo Web (WCAG 2.0). Após a inserção do endereço URL do website, 
a ferramenta gera um resumo apresentando uma escala quantitativa (índice AccessMonitor), os erros encontrados por grau de conformidade (A, AA e AAA), bem como um relatório detalhado do teste realizado. O índice global do AccessMonitor varia de 1 (muito má prática de acessibilidade na Web) a 10 (excelente prática da acessibilidade na Web).

OTAW também é uma ferramenta online automática que verifica o nível de acessibilidade de uma página Web tendo em conta as WCAG 2.o. Os resultados são apresentados em três categorias: (i) "problemas" (são necessárias correções); (ii) "avisos" (é necessária uma revisão por parte de um técnico) e, por fim; (iii) "não revisto" (em que a ferramenta assinala a possibilidade de existir um problema, sendo necessário uma revisão totalmente manual por parte de um técnico para identificar esse problema). Para avaliar a acessibilidade dos websites das AVs e identificar os problemas e avisos, a ferramenta TAW foi utilizada usando os três graus de conformidade (A, AA e AAA).

Tendo sido feita a análise dos websites no mês de fevereiro de 2019, com os resultados obtidos da utilização de ambas as ferramentas, foi construída uma base de dados em SPSS. Para identificar o nível de acessibilidade dos websites, foram utilizadas estatísticas descritivas (média, mínimo, máximo e desvio padrão). Em seguida, para identificar grupos homogéneos (clusters) de AVs de acordo com a acessibilidade dos websites, foi realizada uma análise de clusters hierárquica, utilizando como método de agregação o método Ward, usando as variáveis "Índice global do AccessMonitor", “AccessMonitor erros tipo A", "AccessMonitor erros tipo AA" e "AccessMonitor erros tipo AAA" para criar os clusters. Posteriormente, os clusters identificados foram comparados utilizando testes estatísticos para comparar grupos, especificamente a ANOVA.

\subsection{Apresentação e discussão dos resultados}

\subsubsection{Caracterização da amostra}

Dos 289 registos presentes na base de dados relativos à Região Centro de Portugal, $192(66,44 \%)$ têm endereço Website associado, enquanto 41 registos (14,19 \%) não têm website associado, mas possuem página de Facebook, e 56 (19,38\%.) não têm nem Website nem Facebook associado. Contudo, após a pesquisa, 12 websites foram "fundidos" em dois por pertencerem a uma rede de agências que utilizam a mesma plataforma (website), resultando assim numa amostra final de 182 websites para análise. De acordo com a Tabela 1, cerca de $71 \%$ dos websites analisados são de AVs localizadas junto à costa, enquanto 7,7\% localizam-se nas Beiras e Serra da Estrela e Beira Baixa. Tendo em conta o ano de registo, os anos 2011, 2018 e 2013 correspondem aos anos onde se verificou um maior número de registos (83, 23 e 20 respetivamente), enquanto que o ano de 2012 compreende o menor número de registos (apenas 7).

\begin{tabular}{lcc|ccc}
\hline \multicolumn{2}{c|}{ Localização } & \multicolumn{3}{c}{ Registo na plataforma } \\
\hline NUTs III & $\mathbf{N}$ & $\mathbf{\%}$ & Ano & N & $\%$ \\
\hline Beira Baixa & 4 & $2,2 \%$ & 2011 & 83 & $45,60 \%$ \\
\hline Beiras e Serra da Estrela & 10 & $5,5 \%$ & 2012 & 7 & $3,85 \%$ \\
\hline Médio Tejo & 25 & $13,7 \%$ & 2013 & 20 & $10,99 \%$ \\
\hline
\end{tabular}




\begin{tabular}{lcc|ccc}
\hline \multicolumn{2}{c}{ Localização } & \multicolumn{3}{c}{ Registo na plataforma } \\
\hline NUTs III & $\mathbf{N}$ & $\mathbf{\%}$ & Ano & N & \% \\
\hline Oeste & 36 & $19,8 \%$ & 2014 & 9 & $4,95 \%$ \\
\hline Região de Aveiro & 22 & $12,1 \%$ & 2015 & 11 & $6,04 \%$ \\
\hline Região de Coimbra & 37 & $20,3 \%$ & 2016 & 13 & $7,14 \%$ \\
\hline Região de Leiria & 34 & $18,7 \%$ & 2017 & 16 & $8,79 \%$ \\
\hline Região Viseu - Dão Lafões & 14 & $7,7 \%$ & 2018 & 23 & $12,64 \%$ \\
\hline Total & $\mathbf{1 8 2}$ & $\mathbf{1 0 0 \%}$ & Total & $\mathbf{1 8 2}$ & $\mathbf{1 0 0} \%$ \\
\hline
\end{tabular}

Tabela 1 - Número de AVs por localização e por ano de registo na plataforma

\subsubsection{Acessibilidade dos websites}

Tendo em conta os resultados obtidos no AccessMonitor (Tabela 2), o valor mínimo obtido do índice de acessibilidade global dos websites das AVs foi de 2,8, enquanto a pontuação máxima verificada foi de 7,3 . No entanto, a medida do índice global de acessibilidade dos websites das agências de viagens na Região Centro de Portugal ficou nos 4,77 .

\begin{tabular}{llllll}
\hline Resultados do AccessMonitor & N & Média & $\begin{array}{l}\text { Desv. } \\
\text { Padrão }\end{array}$ & Mínimo & Máximo \\
\hline Índice Global AccessMonitor & 182 & 4,77 & 0,982 & 2,8 & 7,3 \\
\hline Erros nível A & 182 & 7,14 & 3,090 & 0 & 14 \\
\hline Erros nível AA & 182 & 1,03 &, 783 & 0 & 3 \\
\hline Erros nível AAA & 182 & 1,57 &, 849 & 0 & 3 \\
\hline Total de Erros (A + AA + AAA) & 182 & 9,74 & 3,696 & o & 18 \\
\hline
\end{tabular}

Tabela 2 - Resultados globais do AccessMonitor

Estes resultados revelam que o nível de acessibilidade dos websites analisados situa-se entre o nível baixo e o mediano, comprovando os resultados de outros estudos (usando também o AccessMonitor), nomeadamente, o estudo da Unidade ACESSO (2013), sobre a acessibilidade dos websites dos estabelecimentos de ensino superior em Portugal, na qual, obteve um índice global de 5,3 e a análise (cerca de 78 páginas contabilizadas) da Escola Superior de Educação Social que obteve um índice global de 5,7 (IPL, 2018). Por fim, a análise efetuada pela Fundação para os Estudos e Formação Autárquica (com um total de 74 páginas contabilizadas) destaca-se por apresentar um índice global ainda mais baixo, na ordem dos 2,8 (CEFA, 2012).

Em média, foram detetados 9,74 erros em cada website, tendo sido obtido um máximo de 18 erros e o mínimo igual a zero (Tabela 2). Quanto ao grau de conformidade (A, AA e AAA), o destaque vai para os erros de grau de conformidade A que apresenta um máximo de 14 erros (e mínimo igual a zero) com uma média de 7,14 erros por website analisado, o que significa que os websites analisados não cumprem o mínimo exigido em termos 
de acessibilidade. No que diz respeito aos erros de grau de conformidade AA e AAA, verifica-se em ambos um máximo de 3 erros (e mínimo igual a zero), sendo que, o grupo AAA ostenta uma média superior com 1,57 erros por website analisado, enquanto que a do grupo AA é de 1,03 erros. Em termos globais, dos 182 websites analisados, 99,45\% têm erros de prioridade A, 74,73\% têm de AA e 86,26\% têm erros de nível AAA. Assim, em termos de conformidade, pode-se concluir que apenas $0,55 \%$ (equivalente apenas a um website) dos websites da amostra estão conformes com o nível 'A' das WCAG 2.o, revelando que todos os restantes 181 websites não alcançaram o número mínimo de conformidade (nível A). Estes resultados vão novamente ao encontro da realidade encontrada pela Unidade ACESSO (2013), no seu estudo da acessibilidade dos websites de estabelecimentos de ensino superior em Portugal, na qual, apenas o,8\% da amostra estava conforme o nível A e 99,2\% dos websites apresentavam erros de nível A. Por outro lado, tanto na análise da Escola Superior de Educação Social (IPL) como na análise da Fundação para os Estudos e Formação Autárquica (CEFA), verificou-se que 100\% das páginas falharam nos testes de nível A, ou seja, a conformidade do website com o nível A, é de o\% (CEFA, 2012; IPL, 2018).

Tendo em conta que o valor do índice global do AccessMonitor varia entre 1 e 10, os resultados obtidos (Tabela 3) indicam que um grande número dos websites das agências de viagens (63,74\%), seguem uma "prática regular" (índice entre 4 e 5), enquanto 25,27\% dos websites enquadram-se numa "má prática" (índice entre 2 e 3).

\begin{tabular}{lll}
\hline Índice Global AccessMonitor & $\mathbf{N}$ & $\mathbf{\%}$ \\
\hline Muito má prática [0-2[ & $\mathrm{o}$ & $\mathrm{0}$ \\
\hline Má prática [2-3[ & 46 & 25,27 \\
\hline Prática regular [4-5[ & 116 & 63,74 \\
\hline Boa prática [6-7[ & 20 & 10,99 \\
\hline Muito boa prática [8-9[ & $\mathrm{o}$ & $\mathrm{0}$ \\
\hline Excelente prática [10[ & $\mathrm{o}$ & $\mathrm{o}$ \\
\hline
\end{tabular}

Tabela 3 - Acessibilidade dos websites das Agências de Viagens com base no Índice Global do AccessMonitor

Apenas um número limitado de websites (10,99\%) pode classificar-se como "boa prática" (índice entre 6 e 7) e não existem websites passiveis de classificação "muito boa prática" (índice entre 8 e 9) ou com "excelente prática".

Relativamente ao tipo de erros que o AccessMonitor identificou nos testes realizados em cada website, a maioria varia entre o e 2 erros, porém destacam-se: (i) a marcação de formulários (nível A); (ii) marcação de links, menus e textos dos links (nível AAA); (iii) links para contornar blocos de informação (nível A) e; ainda o (iv) standard $\mathrm{W}_{3} \mathrm{C}$ (Html + CSS) (nível A).

A Marcação de formulários (nível A) apresenta um máximo de 6 erros e média de 1,62 que corresponde ao critério de sucesso "Em Entrada" (3.2.2) do WCAG 2.0, enquanto que a Marcação de links, menus e textos dos links (nível AAA) tem um máximo de 3 erros e média de 0,79. Ainda assim, é de destacar os erros relacionados com "Links 
para contornar blocos de informação" (nível A) e "Standard W3C (Html + CSS)" (nível A), que apresentam as médias mais elevadas de erros (a seguir ao erro "Marcação de formulários”) com 1,48 e 1,27 respetivamente.

\subsubsection{Heterogeneidade na acessibilidade dos websites}

A identificação de grupos homogéneos foi realizada através de uma análise de clusters hierárquica, utilizando como método de agregação o método Ward, usando as variáveis "Índice global do AccessMonitor", “AccessMonitor erros tipo A”, “AccessMonitor erros tipo AA" e "AccessMonitor erros tipo AAA" para criar os clusters. Para identificar o número de clusters a reter, foram analisados o dendograma e a tabela de aglomeração. Com base nesta análise, a solução mais adequada seria a de 3 clusters. Os clusters foram comparados utilizando a ANOVA (Tabela 4), sendo que, os prossupostos deste teste foram analisados (normalidade e homoscedasticidade) tendo-se verificado que, para todas as variáveis, era possível a sua utilização.

\begin{tabular}{|c|c|c|c|c|c|c|}
\hline \multirow[t]{3}{*}{ Componentes } & $\begin{array}{l}\text { Total da } \\
\text { amostra }\end{array}$ & $\begin{array}{l}\text { Cluster } 1 \\
\text { "websites } \\
\text { com menor } \\
\text { acessibilidade" }\end{array}$ & $\begin{array}{l}\text { Cluster } \mathbf{2} \\
\text { "websites com } \\
\text { moderada } \\
\text { acessibilidade" }\end{array}$ & $\begin{array}{l}\text { Cluster } 3 \\
\text { "websites } \\
\text { com maior } \\
\text { acessibilidade" }\end{array}$ & \multicolumn{2}{|l|}{ ANOVA } \\
\hline & $\mathrm{N}=180$ & $\mathrm{~N}=61(33,5 \%)$ & $\mathrm{N}=61(33,5 \%)$ & $\mathrm{N}=60(33 \%)$ & \multirow{2}{*}{$\mathbf{F}$} & \multirow{2}{*}{ p-value } \\
\hline & Média & Média & Média & Média & & \\
\hline Índice Global & 4,775 & $3,787^{1}$ & $5,123^{2}$ & $5,425^{3}$ & 100.527 & 0.000 \\
\hline erro $A$ & 7,14 & $10,23^{2}$ & $5,77^{1}$ & $5,40^{1}$ & 92.484 & 0.000 \\
\hline erro $A A$ & 1,03 & $1,66^{2}$ & $0,74^{1}$ & $0,68^{1}$ & 43,582 & 0.000 \\
\hline erro $A A A$ & 1,57 & $1,80^{2}$ & $2,30^{3}$ & $0,58^{1}$ & 232,537 & 0.000 \\
\hline
\end{tabular}

Legenda: Subconjuntos homogéneos - 1, 2, 3.

Tabela 4 - Comparação de Clusters do AccessMonitor usando a ANOVA.

O Cluster $1(\mathrm{~N}=61)$ - “websites com menor acessibilidade", tem uma representatividade de 33,5\% da amostra total e contém os websites das AVs e OTs com a média mais baixa do Índice global do AccessMonitor (3,789), destacando-se também, os websites com o maior número de erros nos três níveis de conformidade (A, AA, AAA), com especial destaque para o grau de exigência mais baixo (nível de conformidade A) que apresenta uma média de 10,23 erros por teste efetuado. Com igual representatividade (33,5 \%), o Cluster $2(\mathrm{~N}=61)$ - "websites com moderada acessibilidade", integra os websites das AVs e OTs com média positiva $(5,123)$ do Índice global do AccessMonitor e também onde se encontram os websites com o maior número de erros do tipo AAA (média de 10,23). Por fim, o Cluster 3 - "websites com maior acessibilidade", tem uma representatividade de $33 \%(\mathrm{~N}=60)$ da amostra total. Nele estão presentes os websites das AVs e OTs com a melhor pontuação do Índice global do AccessMonitor (média de 5,425), assim como, os websites com a menor média de erros identificados quer do nível de conformidade AA $(0,68)$ quer do nível de conformidade AAA $(0,58)$.

Todavia, por forma a assinalar outras características dos clusters, efetuou-se a comparação pelo tipo de problemas (Tabela 5) e avisos (Tabela 6) identificados pelo 
TAW, tendo em conta os 4 princípios das WCAG (Percetível, Operável, Compreensível e Robusto). Os princípios 'Percetível' e 'Robusto' destacam-se por apresentarem uma média superior de erros identificados tanto ao nível de problemas como de avisos. Relativamente aos problemas, os mesmos princípios destacam-se igualmente nesta análise, apresentando as médias mais altas e enquadrando-se no Cluster 1 - "websites com menor acessibilidade”, ou seja, os websites pertencentes a este cluster apresentam mais falhas nestes componentes, que devido à sua relevância, a sua ausência diminui a acessibilidade de uma página web.

\begin{tabular}{|c|c|c|c|c|c|c|}
\hline \multirow[t]{3}{*}{ Princípios } & $\begin{array}{l}\text { Total da } \\
\text { amostra }\end{array}$ & $\begin{array}{l}\text { Cluster } \mathbf{1} \\
\text { "websites } \\
\text { com menor } \\
\text { acessibilidade" }\end{array}$ & $\begin{array}{l}\text { Cluster } 2 \\
\text { "websites com } \\
\text { moderada } \\
\text { acessibilidade" }\end{array}$ & $\begin{array}{l}\text { Cluster } \mathbf{3} \\
\text { "websites } \\
\text { com maior } \\
\text { acessibilidade" }\end{array}$ & \multicolumn{2}{|c|}{ ANOVA } \\
\hline & $\mathrm{N}=182$ & $\mathrm{~N}=61(33,5 \%)$ & $\mathrm{N}=61(33,5 \%)$ & $\mathrm{N}=60(33 \%)$ & \multirow{2}{*}{$\mathbf{F}$} & \multirow{2}{*}{ p-value } \\
\hline & Média & Média & Média & Média & & \\
\hline Percetível & 41,70 & $85,49^{2}$ & $20,74^{1}$ & $18,50^{1}$ & 27,082 & 0.000 \\
\hline Operável & 22,19 & $32,10^{2}$ & $21,10^{1,2}$ & $13,22^{1}$ & 7,311 & 0.001 \\
\hline Compreensível & 21,21 & $52,34^{2}$ & $4,28^{1}$ & $6,77^{1}$ & 18,924 & 0.000 \\
\hline Robusto & 36,84 & $67,66^{2}$ & $22,66^{1}$ & $19,93^{1}$ & 15,724 & 0.000 \\
\hline
\end{tabular}

Legenda: Subconjuntos homogéneos - 1, 2, 3 .

Tabela 5 - Comparação de Clusters em termos de problemas identificados através do TAW usando a ANOVA.

Contrariamente ao esperado, os problemas relacionados com princípio Compreensível, detém a média mais baixa no Cluster 2 - "websites com moderada acessibilidade", em vez de se verificar no Cluster 3 - "websites com maior acessibilidade", visto que, nos restantes princípios apresenta sempre a média mais baixa comparativamente com os outros clusters. Este resultado pode dever-se ao facto de o cluster 2 conter um grande número de websites em que a informação e a interface do utilizador são mais compreensíveis do que os restantes websites pertencentes aos clusters 1 e 3 .

\begin{tabular}{|c|c|c|c|c|c|c|}
\hline \multirow[t]{3}{*}{ Princípios } & $\begin{array}{l}\text { Total da } \\
\text { amostra }\end{array}$ & $\begin{array}{l}\text { Cluster } 1 \\
\text { "websites } \\
\text { com menor } \\
\text { acessibilidade" }\end{array}$ & $\begin{array}{l}\text { Cluster } 2 \\
\text { "websites com } \\
\text { moderada } \\
\text { acessibilidade" }\end{array}$ & $\begin{array}{l}\text { Cluster } \mathbf{3} \\
\text { "websites } \\
\text { com maior } \\
\text { acessibilidade" }\end{array}$ & \multicolumn{2}{|c|}{ ANOVA } \\
\hline & $\mathrm{N}=182$ & $\mathrm{~N}=61$ & $\%=33,5$ & $\mathrm{~N}=61$ & \multirow{2}{*}{$\mathbf{F}$} & \multirow{2}{*}{ p-value } \\
\hline & Média & Média & Média & Média & & \\
\hline Percetível & 105,62 & $150, \mathrm{OO}^{2}$ & $105,67^{1,2}$ & $60,45^{1}$ & 5,077 & 0.007 \\
\hline Operável & 38,76 & $60,72^{2}$ & $34,84^{1}$ & $20,43^{1}$ & 15,364 & 0.000 \\
\hline Compreensível & 20,60 & $30,36^{2}$ & $18,87^{1}$ & $12,43^{1}$ & 17,271 & 0.000 \\
\hline Robusto & 77,08 & 69,92 & 106,75 & 54,18 & 1,541 & 0.217 \\
\hline
\end{tabular}

Legenda: Subconjuntos homogéneos - 1, 2, 3 .

Tabela 6 - Comparação de Clusters em termos de avisos identificados através do TAW usando a ANOVA. 
Analogamente, também os princípios 'Percetível' e 'Robusto' apresentam as médias mais altas em termos de avisos identificados (Tabela 6). Contudo, o Cluster 2 detém uma média superior no princípio 'Robusto', comparativamente com os restantes clusters, podendo significar que o conteúdo dos websites presentes a este cluster não é robusto o suficiente para poder ser interpretado de forma fiável.

\subsubsection{Recomendações para melhorar a acessibilidade dos websites}

Para um conteúdo Web mais acessível, os websites devem tentar cumprir com os requisitos recomendados pelas WCAG. Porém, tendo em conta os resultados deste estudo, é possível identificar as principais falhas de acessibilidade dos websites das AVs e OTs da Região Centro. Neste sentido, a marcação de formulários (nível A) está relacionada com a falha no critério "Em Entrada" (3.2.2) do WCAG 2.0, que diz respeito ao objetivo que visa disponibilizar um mecanismo para, explicitamente, o utilizador efetuar um pedido de mudança de contexto. Em termos práticos, o sucesso deste critério permite ajudar os utilizadores com incapacidades visuais, ou com limitações cognitivas, avisando-os previamente sobre possíveis alterações de contexto (W3C, 2018).

$\boldsymbol{A}$ Marcação de links, menus e textos dos links (nível AAA) relaciona-se com a "finalidade do link" (2.4.9 - WCAG) e ocorre quando um link é composto apenas por conteúdo não textual, como por exemplo, uma imagem, que deve ser sempre acompanhada de uma legenda (i.e. um equivalente alternativo textual), caso contrário o conteúdo poderá ser ignorado pelas tecnologias de apoio (FCT, 2012) condicionando, assim, o acesso a esta informação por parte de um determinado segmento de PCI. Caso este requisito se cumpra, ajuda ( $\left.\mathrm{W}_{3} \mathrm{C}, 2018\right)$ :

- As pessoas com deficiências motoras, permitindo-lhes ignorar as páginas Web que não lhes interessam, evitando os batimentos de tecla necessários para aceder ao referido conteúdo e, em seguida, regressar ao conteúdo atual;

- As pessoas com limitações cognitivas não ficam desorientadas devido à navegação adicional em conteúdos que não lhes interessam;

- As pessoas com incapacidades visuais beneficiam por não perder a sua localização no conteúdo quando regressam à página original.

Assim sendo, podem aplicar-se algumas técnicas consideradas como sendo boas práticas (W3C, 2018), por forma a colmatar as principais falhas identificadas pelas ferramentas automáticas, nomeadamente: (i) fornecer alternativas de texto para qualquer conteúdo não textual (para que se possa alterar para outros formulários de acordo com o que as pessoas precisam, como impressão em grande, braille, fala, símbolos ou linguagem mais simples); (ii) maximizar a compatibilidade com dispositivos atuais e futuros, incluindo tecnologias de apoio (iii) fornecer um mecanismo para solicitar uma atualização do conteúdo em vez de atualizar automaticamente.

\section{Conclusões, limitações e trabalho futuro}

O presente trabalho, que teve como principal objetivo conduzir um estudo ao nível da acessibilidade dos Websites das AVs e OTs localizados na Região Centro de Portugal, usando, para o efeito, duas ferramentas de avaliação automática, revelou, que os websites analisados apresentam problemas em termos de acessibilidade. Os resultados 
do AccessMonitor permitem classificar os websites como seguindo uma "Prática Regular”, enquanto que os resultados da ferramenta TAW demonstram um maior nível de incidentes nos princípios: 'Percetível' e 'Robusto', sendo o Compreensível o princípio com menor média de erros identificados. Estes resultados corroboram os resultados do estudo de Vila, González \& Darcy (2018), que analisou os websites oficiais de turismo dos países do Norte da Europa, observando que os princípios 'Percetível' e 'Robusto' foram os mais críticos em termos de incidentes registados, com implicações diretas nos utilizadores com incapacidades visuais e limitações cognitivas, bem como na conexão com eventuais tecnologias de apoio. Os resultados do presente estudo, estão igualmente em linha com os dos estudos de Shi (2006) e de Mills, Han e Clay (2008) que concluíram que os websites analisados falharam em pelo menos um critério das WCAG, dificultando o acesso e a correta navegação pelos websites por parte dos utilizadores com algum tipo de incapacidade ou necessidade especial.

Tendo em conta o grau de conformidade, ambas as ferramentas utilizadas na análise detetaram um maior número de erros, problemas e avisos no nível A (grau de conformidade mais baixo), seguindo-se o nível AAA (grau de conformidade mais alto) e por último o nível AA. Na verdade, isto revela que muitos dos websites não preenchem os requisitos básicos da acessibilidade da web, constituindo ou aumentando assim, uma barreira impeditiva da utilização dos websites por parte das PCI. Estes resultados são concordantes com os encontrados no estudo de AkgüL e Vatansever (2016), que concluíram que a grande maioria dos websites analisados - do governo Turco - não cumpriam os níveis mínimos dos requisitos de acessibilidade Web. Embora se possa concluir que os websites apresentam um nível de acessibilidade medíocre, existem diferenças entre eles (websites com menor acessibilidade, websites com moderada acessibilidade e websites com maior acessibilidade) no que toca ao tipo de erros (nível A, AA, AAA) e princípios (Percetível, Operável, Compreensível e Robusto).

Dada a ausência de trabalhos científicos sobre esta temática, nomeadamente no contexto das AVs e OTs, considera-se que este estudo apresenta relevantes contributos teóricos para a comunidade científica. Em termos práticos os contributos prendemse, essencialmente, com a possibilidade de: (i) perceber o estado da acessibilidade dos websites das AVs e OTs da Região Centro de Portugal; (ii) identificar as principais falhas na acessibilidade dos websites; (iii) identificar as diferenças existentes na acessibilidade dos websites e também (iii) identificar algumas ações que podem ser implementadas para aumentar o nível de acessibilidade dos websites das AVs e OTs.

Apesar dos relevantes contributos deste estudo, algumas limitações podem ser identificadas, uma vez que as ferramentas utilizadas para avaliar os websites das AVs e OTs da Região Centro de Portugal apenas avaliam o WCAG 2.0 e, apesar de serem automáticas (o que facilita a obtenção de resultados) estão restringidas a verificar a presença ou ausência de um determinado atributo ou elemento (por exemplo: texto alternativo ou legendas), ignorando assim, a visão e perceção das pessoas que vivenciam essas situações. Também se pode considerar uma limitação, a natureza dinâmica dos conteúdos dos websites, na qual, pode influenciar os problemas detetados de acordo com o período temporal em que se aplica a análise com as ferramentas. Para colmatar algumas destas limitações considera-se relevante no futuro desenvolver estudos sobre as seguintes temáticas (i) avaliar os websites das AVs com outras ferramentas para 
comparação de resultados; (ii) replicar o estudo em outras regiões de Portugal ou até mesmo elaborar um estudo a nível nacional e ainda (iii) efetuar uma avaliação humana, de forma a perceber as dificuldades que se encontram na aquisição de serviços nos intermediários relacionadas com a acessibilidade da informação que é fornecida pelos intermediários e comparar estes com os resultados obtidos com as ferramentas de avaliação automáticas.

\section{Acknowledgment}

"This work was also financially supported by the project POCI-01-0145-FEDER-030376, funded by FEDER, through COMPETE2020 - Programa Operacional Competitividade e Internacionalização (POCI), and by national funds (OE), through FCT/MCTES.”

\section{Referências}

Allan, M. (2015). Accessible tourism in Jordan: travel constrains and motivations. European Journal of Tourism Research, 10, 109-119.

AkgÜL, Y., \& Vatansever, K. (2016). Web Accessibility Evaluation of Government Websites for People with Disabilities in Turkey. Journal of Advanced Management Science, (April), 201-210. https://doi.org/10.12720/joams.4.3.201-210

Bowtell, J. (2015). Assessing the value and market attractiveness of the accessible tourism industry in Europe: a focus on major travel and leisure companies. Journal of Tourism Futures, 1(3), 203-222. https://doi.org/10.1108/JTF-03-2015-0012

Buhalis, D., \& Michopoulou, E. (2011). Information-enabled tourism destination marketing: addressing the accessibility market. Current Issues in Tourism, 14(2), 145-168. https://doi.org/10.1080/13683501003653361

Buhalis, D., \& Darcy, S. (2010). Accessible Tourism: Concepts and Issues. Channel View Publications.

Card, J. A., Cole, S. T., \& Humphrey, A. H. (2006). A comparison of the Accessibility and Attitudinal Barriers Model: Travel providers and travelers with physical disabilities. Asia Pacific Journal of Tourism Research, 11(2), 161-175. https://doi. org/10.1080/10941660600727566

Carvalho, R. M., Lopes, P. P. F., Alexandre, I., \& Alturas, B. (2016). Qualidade dos sítios Web da Administração Pública Portuguesa. Revista Ibérica de Sistemas e Tecnologias de Informação. 20, 78-98. http://dx.doi.org/10.17013/risti.20.78-98

CEFA (2012). AccessMonitor - Fundação para os Estudos e Formação Autárquica (CEFA). Obtido de http://www.acessibilidade.gov.pt/accessmonitor/wcag20/?sid=1517

Costa, J., Salvado, A., \& Da Costa, C. (2014). Cocriação de valor : uma perspetiva das agências de viagens independentes. Revista turística \& Desenvolvimento, 104, (2122), 35-50.

Darcy, S. (2006), Setting a Research Agenda for Accessible Tourism, Sustainable Tourism for Cooperative Research Centre, Gold Coast. 
Darcy, S., \& Dickson, T. J. (2009). A whole-of-life approach to tourism: The case for accessible tourism experiences. Journal of Hospitality and Tourism Management, 16(1), 32-44. https://doi.org/10.1375/jhtm.16.1.32

Gillovic, B., \& McIntosh, A. (2015). Stakeholder perspectives of the future of accessible tourism in New Zealand. Journal of Tourism Futures, 1(3), 223-239. https://doi. org/10.1108/JTF-04-2015-0013

IPL (2018). AccessMonitor - Escola Superior de Comunicação Social, IPL. Obtido de http://www.acessibilidade.gov.pt/accessmonitor/wcag20/?sid=2841\&e=A

Kanellou, D. (2000). The new role of intermediaries in travel and tourism distribution channels. Institute for Prospective Technology Studies. Obtido de http://www. researchgate.net/profile/Despina_Kanellou/publication/259694663_The_New_ Role_of_Intermediaries_in_Travel_and_Tourism_Distribution_Channels/ links/oob4952d5e3918fa7boooooo.pdf

Kastenholz, E., Eusébio , C., \& Figueiredo, E. (2015). Contributions of tourism to social inclusion of persons with disability. Disability and Society, 30, 1259-1281. doi:10.1 080/09687599.2015.1075868

Kim, S. E., \& Lehto, X. Y. (2012). The voice of tourists with mobility disabilities: insights from online customer complaint websites. International Journal of Contemporary Hospitality Management, 24(3), 451-476.

Kiruthika, D., \& Bindu, V. (2016). Accessible Tourism: Determinants and Constraints; A Demand Side Perspective. IOSR Journal of Business and Management, 18(9), 01-08. doi:10.9790/487X-1809010108

Kuzma, J. M., Yen, D., \& Oestreicher, K. (2009). Global e-government Web Accessibility: An Empirical Examination of EU, Asian and African Sites.

Law, R., Leung, K., \& Wong, R. (2004). The impact of the Internet on travel agencies. International Journal of Contemporary Hospitality Management, 105(16), 100-107.

Lee, B. Y., \& Cranage, D. A. (2017). Service failure of intermediary service: impact of ambiguous locus of control. Journal of Travel and Tourism Marketing, 34(4), 515530. https://doi.org/10.1080/10548408.2016.1208785

Lin, C. T., Lee, C., \& Chen, W.-Y. (2009). Using fuzzy analytic hierarchy process to evaluate service performance of a travel intermediary. The Service Industries Journal, 29(3), 281-296. https://doi.org/10.1080/02642060701846762

Loi, K. I., \& Kong , W. H. (2017). Tourism for All: Challenges and Issues Faced by People with Vision Impairment. Tourism Planning \& Development, 14(2), 181-197. https://doi:10.1080/21568316.2016.1204357

Luis, C., Rocha, A., \& Marcelino, M. J. (2017). Acessibilidade em Ambientes Virtuais de Aprendizagem. RISTI - Revista Ibérica de Sistemas e Tecnologias de Informação, (25), 54-65. http://dx.doi.org/10.17013/risti.25.54-65 
Lyu, S. O. (2017). Which accessible travel products are people with disabilities willing to pay more?: A choice experiment. Tourism Management, 59, 404-412. Obtido em 3 de Janeiro de 2017, de http://ac.els-cdn.com/So261517716301625/1-s2.oSo261517716301625-main.pdf?_tid=d9af7fde-d46a-11e6-aa8coooooaabofo1\&acd nat=1483746786_16110e9a6ff3ffe5a7aad451e3ad2691.

Mayol, J., Nadal, G. F., \& Coll, A. B. (2011). Análisis y procedimiento de mejora de la accesibilidad web. RISTI - Revista Ibérica de Sistemas e Tecnologias de Informação, (7), 61-73.

Melian , A., Prats, L., \& Coromina, L. (2016). The perceived value of accessibility in religious sites - do disabled and non-disabled travellers behave differently? Tourism Review, 71(2), 105-117. http://dx.doi.org/10.1108/TR-11- 2015-0057

Mesquita, S., \& Carneiro, J. M. (2016). Accessibility of European museums to visitors with visual impairments. Disability \& Society, 31(3), 373-388. doi:10.1080/09687 599.2016.1167671

Michopoulou, E., \& Buhalis, D. (2013). Information provision for challenging markets: the case of the accessibility requiring market in the context of tourism. Information \& Management, 5O(5), 229-239. https://doi.org/10.1016/j.im.2013.04.001

Mills,J.,Han,J., \&Clay,J.(2008).Accessibilityofhospitalityand tourism websites.Cornell Hospitality Quarterly, 49(1), 28-41. https://doi.org/10.1177/1938965507311499

Özogul, G., \& Baran, G. G. (2016). Accessible tourism: the golden key in the future for the specialized travel agencies. Journal of Tourism Futures, 2(1), 79-87. https:// doi.org/10.1108/JTF-03-2015-0005

Ozturk, Y., Yayli, A., \& Yesiltas, M. (2008). Is the Turkish tourism industry ready for a disabled customer's market?: Theviews of hotel and travel agency managers. Tourism Management, 29(2), 382-389. https://doi.org/10.1016/j.tourman.2007.03.011

Pinho, S. (2015). Turismo Acessível : Oportunidades e Desafios para as unidades hoteleiras de Portugal. https://doi.org/10.13140/RG.2.2.21889.45928

Schitko, D., \& Losekoot, E. (2012). An investigation of the attitudes of travel and tourism intermediaries to mature travellers. Journal of Tourism, 13(1), 61-78. https://doi. $\operatorname{org} / 10.1016 / \mathrm{j}$.

Shi, Y. (2006). The accessibility of Queensland visitor information centres' websites. Tourism Management, 27(5), 829-841. https://doi.org/10.1016/j. tourman.2005.05.012

Takeda, K., \& Card, J. A. (2002). U.S. Tour Operators and Travel Agencies: Barriers Encountered When Providing Package Tours to People Who Have Difficulty Walking. Journal of Travel \& Tourism Marketing, 12(1), 47-61. https://doi. org/10.1300/Jo73v12no1_03

United Nations (2007). Convention on the Rights of Persons with Disabilities and Optional Protocol. https://doi.org/10.1038/nmat3357 
Unidade ACESSO (2013). Estudo sobre o estado da Acessibilidade dos sítios Web dos estabelecimentos de ensino superior. Obtido de http://www.acessibilidade.gov.pt/ estudos/2013_ensinosuperior/es2013.html\#n1

UNWTO (1980). Manila Declaration on World Tourism. Obtido de https://www.eunwto.org/doi/pdf/10.18111/unwtodeclarations.1980.6.4.1

UNWTO (1999). Global Code of Ethics for Tourism.

UNWTO (2016a). Accessible Tourism for All: An Opportunity within Our Reach. https:// doi.org/10.18111/9789284417919

UNWTO (2016b). Compilation of UNWTO Recommendations 1975-2015.

UNWTO (2016c). Manual on Accessible Tourism for All: Principles, Tools and Best Practices Module I: Accessible Tourism - Definition and Context. Madrid.

Vila, T., González, E., \& Darcy, S. (2018). Accessible tourism online resources: a Northern European perspective. Scandinavian Journal of Hospitality and Tourism, o(o), 1-17. https://doi.org/10.1080/15022250.2018.1478325

Vila, T., González, E., \& Darcy, S. (2017). Website accessibility in the tourism industry: an analysis of official national tourism organization websites around the world. Disability and Rehabilitation, o(o), 1-12. https://doi.org/10.1080/09638288.20 17.1362709

W3C. (2018). Web Content Accessibility Guidelines (WCAG) 2.1. Obtido de https:// www.w3.org/TR/2018/REC-WCAG21-20180605/ 